\title{
Planeamento da Carreira, Comportamento e Rendimento Académico no Ensino
}

\author{
Básico

\section{Career Planning, Behaviour and Academic Performance in Basic Education}

\author{
Maria Taveira, Adriana Azevedo, Íris Oliveira \\ Universidade do Minho
}

\begin{abstract}
Resumo
O planeamento de carreira estimula sentimentos de competência pessoal que habilitam a lidar com a carreira e o futuro. Analisa-se a relação simultânea entre planeamento da carreira e comportamento e desempenho académico. Participaram 442 alunos do $5^{\circ}$ ano $(231,52.2 \%$ rapazes), com idades entre nove e 13 anos ( $M=10.23, D P$ $=0.49$ ). Utilizou-se um questionário de identificação e a subescala planeamento da Childhood Career Development Scale. Os resultados evidenciam dois perfis de carreira: o construtivo, com mais planeamento, comportamento e resultados académicos positivos; o vulnerável, com o planeamento mais baixo, problemas comportamentais, mais dificuldades na aprendizagem e retenções.

Palabras chave: desenvolvimento de carreira, infância, planeamento da carreira, desempenho académico.
\end{abstract}

\begin{abstract}
Career planning stimulates personal competence and coping with career and future. We analyse the simultaneous relationship between career planning and academic behaviour and performance. Participants were 446 students from the $5^{\text {th }}$ year $(231,52.2 \%$ boys $)$, aged nine to 13 years old $(M=10.23, S D=0.49)$. A demographic questionnaire and the planning subscale of the Childhood Career Development Scale were used. Results evidenced two different career profiles: the constructive, with higher planning and positive outcomes in academic behaviour and performance; the vulnerable, with lower planning, behavioural problems, more learning difficulties, and school retention. Keywords: career development, childhood, career planning, academic performance.
\end{abstract}

O desenvolvimento vocacional ou de carreira traduz-se num processo dinâmico de aquisição de competências e aprendizagens, no qual o indivíduo deve assumir um papel ativo acerca do mundo de trabalho, ao longo do ciclo vital (Oliveira, 2016; Super, 1990). Apesar da investigação vocacional incidir maioritariamente na adolescência e na adultez, tem-se reconhecido a importância da infância no desenvolvimento vocacional (e. g., Araujo, S., 2002; Araújo, A., 2009; David, 2017; Oliveira, 2016; Taveira, 1999). Neste sentido, a infância (0-14 anos) caracteriza-se como uma etapa de crescimento e mudança, marcada por múltiplas capacidades humanas, como o pensamento e raciocínio, a linguagem e comunicação, a criatividade, a emoção, e o relacionamento interpessoal (Araújo, 2009, p. 3). Em
1996, Herr e Cramer salientam que durante este período assistimos à formação dos primeiros objetivos, ao início da construção da motivação para a realização e das perceções de si como competente (Araújo, 2009). Na mesma linha de pensamento, Gottfredson (1996), Super (1990) e Savickas (2002), mencionam que a infância é uma etapa de desenvolvimento ativo, onde a criança em interação com diversos contextos, desenvolve os primeiros interesses, competências e perspetivas futuras, possibilitando a aquisição da capacidade de autocontrolo e a consciencialização do seu papel interventivo, tanto ao nível académico, como profissional. Neste contexto, Lopes (2004) refere que o desenvolvimento vocacional na infância visa a promoção "de um conjunto de competências a adquirir ao longo da escolaridade, desde os primeiros níveis, funcionando como um fator de prevenção do abandono escolar e, ao mesmo tempo, de afirmação da instrumentalidade da escola na formação de cidadãos" (p. 193), indo ao encontro dos objetivos do Ensino Básico e da Educação em geral. Neste âmbito ainda, Super (1990) nota que o desenvolvimento do planeamento da carreira, na infância, acompanha a capacidade de identificar e resolver problemas, nomeadamente no domínio escolar, e mais tarde, a capacidade de tomar decisões. Assim, é esperado que durante a infância, a criança aumente o controlo das suas ações, reconheça a importância de ser bem-sucedida na escola e integre hábitos de trabalho. Aponta-se, desde logo, para a possível articulação entre processos de carreira e académicos na infância, sendo importante que a criança perceba desde cedo, e progressivamente, a relação que comportamentos académicos podem ter com o seu futuro mais imediato ou a médio prazo. Ainda que sejam escassos os estudos sobre o planeamento da carreira, sobretudo na infância, os estudos de Nazli (2007) e Schultheiss, Palma e Manzi (2005) evidenciam a importância do planeamento da carreira na infância para a promoção do sucesso numa fase mais avançada da vida. Estes estudos salientam a necessidade de investigação que articule variáveis de carreira e académicas que informem a intervenção psicológica em contextos educativos. Neste sentido, o presente estudo pretende analisar em que medida o planeamento da carreira se relaciona com comportamentos e resultados 
académicos positivos, e define perfis de carreira na infância.

\section{Participantes}

A amostra foi recrutada de modo não probabilístico, intencional. Inclui 442 alunos do $5^{\circ}$ ano, 231 (52.2\%) rapazes e $211(47.8 \%)$ raparigas, com idades entre os nove e 13 anos $(M=10.23, D P=.49)$, de quatro escolas públicas, duas no Norte e duas no centro de Portugal. Com base na Classificação Portuguesa das Profissões 2010 (INE, 2011), verifica-se que a maior parte das mães se enquadra na categoria dos trabalhadores de serviços pessoais, de proteção, segurança e vendedores (29.9\%), e dos especialistas das profissões intelectuais e científicas (15.2\%). Em menor número encontram-se representantes do poder legislativo e de órgãos executivos $(0.9 \%)$ e técnicas e profissionais de nível intermédio (4.1\%). Os pais enquadram-se maioritariamente nos trabalhadores qualificados da indústria, construção e artífices (31.9\%), $\mathrm{n}$ os operadores de serviços pessoais, e proteção, segurança e vendedores $(23.1 \%)$, e nos profissionais das forças armadas, e agricultores e trabalhadores qualificados da agricultura, da pesca e da floresta $(0.2 \%$ e $0.5 \%$ respetivamente). As mães e os pais estão, na sua maioria, empregados ( mães $=71.3 \%$; pais $=83.2 \%$ ).

\section{Instrumentos}

Questionário de Identificação (QID; Araújo, 2009; adaptado por Oliveira \& Taveira, 2014). Permitiu recolher dados sócio demográficos (sexo, idade, escola) e académicos (média das classificações escolares do último período letivo, retenções prévias, sinalização pelos professores de dificuldades de aprendizagem e problemas de comportamento, e apoios escolares recebidos no momento da investigação) dos participantes.

Childhood Career Development Scale (CCDS; Schultheiss \& Stead, 2004). Utilizou-se a subescala de planeamento da versão portuguesa da CCDS (Oliveira, 2016), com oito itens (e.g. "Sei que planear é importante"), respondidos numa escala de cinco pontos (1= Discordo Fortemente; 5=Concordo Fortemente), com evidência de consistência interna ( $\alpha$ de Cronbach $=$ .86).

\section{Procedimentos}

O projeto foi aprovado pela Direção Geral da Educação (DGE) e Direções das Escolas envolvidas na investigação. As turmas e os tempos letivos destinados à avaliação foram definidos pelos diretores e psicólogos das escolas. Obtiveram-se os consentimentos informados dos encarregados de educação dos alunos e garantiu-se a participação voluntária destes. A avaliação decorreu entre fevereiro e março de 2013. O QID foi preenchido por investigadoras, para cada aluno, com base em registos escolares, enquanto a CCDS foi administrada no grupo-turma. Garantiu-se a confidencialidade dos dados na sua recolha e análise. Os dados foram tratados com o programa estatístico Statistical Package for Social Sciences (IBM SPSS, Versão 20.0 para Windows). Realizaram-se análises de estatística descritiva (análise de frequências, cálculo de médias e desvios-padrão), uma análise de Clusters para testar a existência de perfis de carreira distintos. Seguindo recomendações da literatura (e.g., Marôco, 2007; Sarstedt \& Mooi, 2014), utilizaramse métodos hierárquicos da análise de clusters e análises gráficas do dendograma para decidir o número de clusters a reter. $\mathrm{O}$ aprofundamento analítico da solução encontrada, recorreu ao método não hierárquico ( $K$ Means Clustering Analysis).

\section{Resultados}

Analisaram-se soluções de dois, três e quatro clusters, tendo-se optado pela solução de dois clusters, a mais coerente na distribuição de casos por cluster. Esta solução foi refinada com o método $K$-Means, com K = 2 e foi também a mais apropriada para a amostra. Contudo, verifica-se disparidade do número de participantes por cluster ( $\mathrm{n}=405$ no primeiro e $\mathrm{n}=37$ no segundo), tendose repetido as análises, após selecionar aleatoriamente casos do primeiro cluster, para obter uma distribuição aleatória mais equilibrada e verificar se os resultados se mantinham. Assim, selecionaram-se aleatoriamente, recorrendo ao comando Random Samples of Cases do SPSS, 38 casos do cluster 1 para equilibrar a amostra face ao cluster $2(\mathrm{n}=38)$. Realizaram-se os mesmos testes estatísticos com a amostra inicial e a aleatória, tendo-se obtido o mesmo padrão de resultados. Apresentam-se os resultados da amostra inicial e aleatória. Para melhor caracterizar cada um dos clusters, foram analisadas as características demográficas dos indivíduos que constituem cada um deles. O quadro 1 apresenta os dados sociodemográficos (área geográfica, sexo e idade) dos participantes por cluster, para cada uma das amostras estudadas.

Quadro 1.

Dados sociodemográficos dos participantes por cluster

O quadro 2 apresenta as médias (centros) da variável intervalar planeamento em cada um dos dois clusters, bem como a estatística T. Não se verificou uma distribuição normal da variável de planeamento. Em consequência, realizaram-se testes paramétricos (Teste T para amostras independentes) e não-paramétricos (Teste de Mann-Whitney) (Martins, 2011). Como os resultados paramétricos e não-paramétricos convergiam, relatam-se

\begin{tabular}{|c|c|c|c|c|c|c|c|c|}
\hline & \multicolumn{4}{|c|}{ Amostra Total } & \multicolumn{4}{|c|}{ Amostra Aleatória } \\
\hline & \multicolumn{2}{|c|}{ Cluster 1} & \multicolumn{2}{|c|}{ Cluster 2} & \multicolumn{2}{|c|}{ Cluster 1} & \multicolumn{2}{|c|}{ Cluster 2} \\
\hline & $n$ & $\%$ & $n$ & $\%$ & $n$ & $\%$ & $n$ & $\%$ \\
\hline Norte & 8,5 & 69.9 & 28 & $\begin{array}{c}75 . \\
7\end{array}$ & $\begin{array}{l}3 \\
4\end{array}$ & $\begin{array}{c}89 . \\
5\end{array}$ & $\begin{array}{l}2 \\
9\end{array}$ & $\begin{array}{c}74 . \\
4\end{array}$ \\
\hline Centro & 122 & 30.1 & 9 & $\begin{array}{c}24 . \\
3\end{array}$ & 4 & $\begin{array}{c}10 . \\
5\end{array}$ & $\begin{array}{l}1 \\
0\end{array}$ & $\begin{array}{c}25 \\
6\end{array}$ \\
\hline Rapariga & 197 & 48.6 & 14 & 37. & 1 & 44. & 1 & 35. \\
\hline Rapazes & 208 & 51.4 & 23 & $\begin{array}{c}62 . \\
2\end{array}$ & $\begin{array}{l}2 \\
1\end{array}$ & $\begin{array}{c}55 . \\
3\end{array}$ & $\begin{array}{l}2 \\
5\end{array}$ & $\begin{array}{c}64 . \\
1\end{array}$ \\
\hline 9 Anos & 2 & 0.5 & - & - & - & - & - & - \\
\hline 10 Anos & 329 & 81.2 & 17 & $\begin{array}{c}45 \\
9\end{array}$ & $\begin{array}{l}2 \\
8\end{array}$ & $\begin{array}{c}73 . \\
7\end{array}$ & $\begin{array}{l}1 \\
9\end{array}$ & $\begin{array}{c}48 . \\
7\end{array}$ \\
\hline 11 Anos & 69 & 17.0 & 14 & $\begin{array}{c}37 . \\
8\end{array}$ & 9 & $\begin{array}{c}23 . \\
7\end{array}$ & $\begin{array}{l}1 \\
4\end{array}$ & $\begin{array}{c}35 . \\
9\end{array}$ \\
\hline 12 Anos & 5 & 1.2 & 5 & $\begin{array}{c}13 . \\
5\end{array}$ & 1 & 2.6 & 5 & $\begin{array}{c}12 \\
8\end{array}$ \\
\hline 13 Anos & - & - & 1 & 2.7 & - & - & 1 & 2.6 \\
\hline Total $(\%)$ & 405 & 100 & 37 & 100 & $\begin{array}{l}3 \\
8\end{array}$ & 100 & $\begin{array}{l}3 \\
9\end{array}$ & 100 \\
\hline
\end{tabular}


os resultados paramétricos. Verificou-se que os alunos do cluster 1 apresentavam níveis significativamente mais elevados de planeamento do que os alunos do cluster 2.

Quadro 2

Centros dos clusters e estatística t no planeamento

\begin{tabular}{lccc}
\hline & \multicolumn{3}{c}{ Centro do Cluster } \\
\hline $\begin{array}{l}\text { Planeamento Amostra Inicial } \\
\text { 3.02 }\end{array}$ & 36.03 & 33.84 & $\mathrm{t}$ \\
$\begin{array}{l}\text { Planeamento Amostra Aleatória } \\
4.20\end{array}$ & 37.76 & 34.00 \\
$\quad$ & & \\
\hline
\end{tabular}

No quadro 3 apresentam-se as médias (centros) das variáveis dicotómicas académicas em cada cluster, e a estatística Phi $(\phi)$ para as retenções, problemas comportamentais e dificuldades de aprendizagem. Os participantes do cluster 1 apresentam significativamente menos retenções, problemas de comportamento e dificuldades de aprendizagem $(M=36.03$ na amostra inicial; $M=37.76$ na amostra aleatória, $t=3.02)$ do que os participantes do cluster $2(\mathrm{M}=33.84$ na amostra inicial; $M=34.00$ na amostra aleatória, $t=4.20$ ).

Quadro 3

Centro dos clusters e estatística Phi $(\phi)$ nas variáveis académicas

\begin{tabular}{cccc}
\hline & Centro Cluster \\
& 1 & 2 & $\phi$ \\
\hline \multicolumn{4}{c}{ Amostra Inicial } \\
Retenções & 0.97 & 0.54 & -.47 \\
$\begin{array}{c}\text { Problemas } \\
\text { comportamentais } \\
\text { Dificuldades }\end{array}$ & 0.85 & 0.05 & -.54 \\
de aprendizagem & 0.91 & 0.14 & -.59 \\
& \multicolumn{4}{c}{ Amostra Aleatória } \\
$\begin{array}{c}\text { Retenções } \\
\text { Problemas }\end{array}$ & 0.97 & 0.56 & -.48 \\
comportamentais & 0.79 & 0.13 & -.66 \\
$\begin{array}{c}\text { Dificuldades } \\
\text { de aprendizagem }\end{array}$ & 0.84 & 0.05 & -.80 \\
& \multicolumn{4}{c}{}
\end{tabular}

Nota. Variáveis académicas codificadas com $0=\operatorname{Sim} 1=$ Não

O cluster 1 caracteriza $91.6 \%$ e $49.4 \%$, respetivamente, da amostra inicial e aleatória. Inclui os alunos com planeamento mais elevado, na amostra, com poucas ou nenhumas retenções prévias, com poucos ou nenhuns registos de problemas comportamentais ou dificuldades de aprendizagem. Este grupo nomeou-se de construtivo. O cluster 2 inclui um grupo reduzido de alunos na amostra inicial, $8.4 \%$, e $50.6 \%$ na amostra aleatória. Pertencem a este grupo, os participantes que exibem valores mais baixos de planeamento, que já reprovaram no seu percurso escolar, com registos de alguns problemas de comportamento e de algumas dificuldades de aprendizagem. Este grupo foi denominado de vulnerável.

\section{Discussão e conclusão}

O objetivo deste estudo foi analisar a relação simultânea entre o planeamento da carreira e variáveis do desempenho académico, de modo a identificar perfis de carreira em estudantes portugueses do $5^{\circ}$ ano de escolaridade. Identificaram-se dois perfis diferenciados de alunos, nomeados de construtivo e vulnerável, com base na teoria e investigação vocacional na infância (e. g., Araújo, A., 2002; Araújo S., 2002; David, 2017; Nazli, 2007; Oliveira, 2016; Super, 1990). O perfil construtivo caracteriza um maior planeamento de carreira e comportamentos e desempenho académicos mais positivos, enquanto o padrão vulnerável caracteriza um menor planeamento e um padrão de vida académica com mais dificuldades e problemas dos alunos, tal como registados pelos docentes, e pior desempenho escolar.

Estes resultados indicam a existência de necessidades distintas de intervenção psicoeducacional nos dois agrupamentos de alunos. E a urgência de articular melhor esta intervenção com intervenções vocacionais precoces, uma vez que um dos objetivos da psicologia vocacional é incentivar as crianças a explorar e a refletir, de modo intencional e progressivo, sobre os seus papeis de vida (e.g., estudante, cidadão) e os diversos contextos de trabalho. Isto será importante, mesmo que a discrepância de número de participantes entre os clusters verificada na amostra inicial - com um numero mais elevado de alunos no perfil construtivo - possa indicar que muitos dos alunos deste estudo adquiriram competências de planeamento que se repercutem no seu sucesso escolar.

Com efeito, as crianças classificadas no perfil construtivo apresentam elevadas competências de planeamento face aos valores da média amostral. Este resultado sugere que estas crianças podem deter mais competências e expectativas de autoeficácia em relação ao seu futuro vocacional. De facto, a infância é um período central para a aprendizagem vocacional e para o desenvolvimento de competências básicas de carreira, como é o caso da exploração, decisão e o planeamento (Oliveira, 2016).

Super (1990) refere que o desenvolvimento do planeamento auxilia a capacidade de identificar e resolver problemas, ou de tomar decisões. Desta forma, torna-se, além de interessante, algo que pode ajudar a criança a atingir os seus objetivos pessoais e a antecipar e preparar as suas atividades. Além disso, é de notar que a transição da escola para o mundo do trabalho resulta de um processo de preparação contínua, ao longo da escolaridade, na qual os pais, em conjunto com a escola e os professores, têm um papel fundamental, nomeadamente, no desenvolvimento de interesses académicos e profissionais, na promoção de escolhas vocacionais e na transmissão de valores de persistência e desempenho educacional e profissional (Oliveira, 2016).

Por sua vez, as crianças com comportamentos favoráveis ao sucesso académico, aumentam os níveis de satisfação com a qualidade e quantidade de informação disponível para a tomada de decisão, e registam níveis de stress favoráveis à exploração e ao planeamento da carreira (Taveira, 1999). Neste estudo, as crianças com um perfil construtivo, apresentam ainda níveis positivos 
em todas as dimensões do desempenho académico, em comparação com a média da amostra, demonstrando estarem a ser capazes de adequar a forma de lidar com eventuais barreiras e desafios específicos nesse domínio de vida. Como esperado, foi possível verificar a existência de uma relação positiva entre o planeamento e as variáveis do desempenho académico.

O segundo grupo de alunos, denominado vulnerável, exibe comportamentos pouco intencionais de planeamento, encontrando-se significativamente abaixo da média global. Araújo (2009) refere que a exploração tem impacto, em idades tardias, no ajustamento académico, formação da identidade, tomada de decisão e no planeamento de carreira. Podemos então mencionar que estas crianças são mais vulneráveis no planeamento de futuras atividades. Estes alunos parecem caracterizarse por crenças negativas relativas a variáveis do desempenho académico, apresentam um percurso académico marcado por dificuldades de aprendizagem e problemas comportamentais, resultando, nalguns casos, em retenções.

A literatura tem demonstrado que é importante implementar programas de intervenção vocacional, de caracter preventivo e promocional, ao longo da escolaridade, articulados com o currículo e com a participação das figuras-chave (e.g., pais e professores) (Oliveira, 2016). Por exemplo, os estudantes do perfil vulnerável poderiam beneficiar de uma intervenção mais abrangente, que os ajudasse a antecipar e a lidar com as barreiras académicas e profissionais que possam existir, bem como de apoios ao estudo, dentro e fora da sala de aula, como por exemplo, recorrer a professores e/ou colegas mentores, que ajudem na realização de determinadas tarefas, no sentido de apoiar o estudo individual e fomentar a sua autorregulação.

Por outro lado, no que respeita aos alunos do perfil construtivo, será importante reforçar estes alunos para manterem e desenvolverem este padrão positivo de carreira ao longo da escolaridade, acompanhando o percurso académico destes alunos, intervindo nas suas abordagens ao estudo e à escola sempre que necessário e proporcionando, de modo sistemático e intencional, oportunidades de desenvolvimento vocacional, e de preparação da carreira/vida, assentes em teoria e investigação da carreira.

Conclui-se que estes resultados podem informar o desenho de intervenções de carreira diferenciadas no ensino básico, de modo a responder de forma ajustada às características de cada grupo de alunos.

\section{Agradecimentos}

Uma primeira apresentação deste trabalho foi realizada em 2017, na Universidade do Minho, pela segunda autora, numa tese de mestrado integrado em Psicologia, integrada no projeto "Trajetórias de desenvolvimento vocacional na infância: Estudo com alunos do ensino básico", financiado pela Fundação para a Ciência e Tecnologia (SFRH/BD/84162/2012), desenvolvido pela terceira autora, ambos sob orientação da primeira autora.

\section{Referências}

Araújo, A. (2009). Antecedentes, dinâmica e consequentes do desenvolvimento vocacional na infância. Tese de Doutoramento não publicada. Universidade do Minho, Braga.

Araujo, S. (2002). Desenvolvimento vocacional na infância. Um estudo exploratório com crianças em idade pré-escolar. Tese de mestrado não publicada. Braga: Universidade do Minho.

Bandura, A., Barbaranelli, C., Caprara, G. V., \& Pastorelli, C. (2001). Self-efficacy beliefs as shapers of children's aspirations and career trajectories. Child Development, 72(1), 187-206.

David, R. (2017). Aspirações vocacionais na infância:Um estudo longitudinal. Tese de doutoramento não publicada. Coimbra: Universidade de Coimbra.

Gottfredson, L. (1996). Gottfredson's theory of circumscription and compromise. In D. Brown, L. Brooks \& Assoc. (Eds.), Career choice and development (pp.179-232). San Fransisco, CA: JosseyBass.

Herr, E. L., \& Cramer, S. H. (1996). Career Guidance and Counseling through the Lifespan: Systematic Approaches. New York: Harper Collins College Publishers.

INE. (2011). Classificação portuguesa das profissões 2010. Lisboa, Portugal.

Lopes, C. (2004). Desenvolvimento Vocacional na infância e prevenção do abandono escolar: contributos dos Serviços de Psicologia e Orientação. In M. C. Taveira (Coord.), Desenvolvimento vocacional ao longo da vida. Fundamentos, princípios e orientações (pp. 191-196). Coimbra: Almedina.

Martins, C. (2011). Manual de análise de dados quantitativos com recurso ao IBM SPSS:Saber decidir, fazer, interpretar e redigir. Braga: Psiquilíbrios Edições.

Marôco, J. (2007). Análise estatística - Com utilização do SPSS (3. ${ }^{\mathrm{a}}$ ed.). Lisboa: Edições Sílabos.

Nazli, S. (2007). Career development in primary school children. Career Development International, 12(5), 446-462. doi:10.1108/13620430710773763

Nurmi, J. E. (1991). How do adolescents see their future? A review of the development of future orientation and planning. Developmental Review, 11(1),1-59. doi:10.1016/0273-2297(91)90002-6

Oliveira, I. M. (2016). Construction and Validation of the Childhood Career Exploration Inventory. Tese de doutoramento não publicada. Universidade do Minho, Braga.

Oliveira, I. \& Taveira, M. C. (2014). Avaliação do desenvolvimento vocacional na infância. Novas Edições Académicas.

Oliveira, I., \& Taveira, M.C. (2016). Desenvolvimento vocacional na infância: Contributos para uma abordagem integradora. In N.L. Pereira-Silva, A.J.G. Barbosa, \& M.C. Rodrigues (Eds.), Pesquisas em desenvolvimento humano e educação, (pp. 355-384). Brasil: Editora CRV. 
Sarstedt, M. \& Mooi, E. (2014). A concise guide to market research: The process, data, and methods using IBM SPSS Statistics. Verlag Berlin, Heidelberg: Springer.

Savickas, M.L. (2002). Revitalizing the study of careers. Journal of Vocational Behavior,61, 381-385. doi:10.1006/jvbe.2002.1880

Schultheiss, D., and Stead, G. B. (2004). Childhood career development scale: scale construction and psychometric properties. Journal of Career Assessment, 12, 113-134. doi:10.1177/1069072703257751

Schultheiss, D., Palma, T. \& Manzi, A. (2005). Career Development in Middle Childhood: A Qualitative Inquiry. The Career Development Quarterly, 53, 246262.doi: 10.1002/j.2161-0045.2005.tb00994.x

Super, D. E. (1990). A life-span, life-space approach to career development. In D. Brown, \& L. Brooks (Eds.), Career choice and development: applying contemporary theories to practice (2. ${ }^{\mathrm{a}}$ ed., pp. 197261). San Francisco, CA: Jossey Bass.

Taveira, M. (1999). Intervenção precoce no desenvolvimento vocacional. Psicologia: teoria, investigação e prática, 4(1), 173-190. 